\title{
Asthma in the emergency room in Canada
}

\section{L'asthme à l'urgence au Canada}

Tn the current issue of the Canadian Respiratory Journal, Rowe et al (pages 25-30 [1]) report the results of a prospective study of an experience with 695 adult asthmatic patients in emergency rooms (ERs) of 20 hospitals across Canada. This study is noteworthy simply because it was completed - it was a 'Herculean' task.

In brief, asthmatic patients who presented to ERs were enrolled after giving informed consent and completing standardized questionnaires. Their course in the ER as well as a follow-up telephone contact was documented. Patients who were admitted as a result of their ER visit were compared with

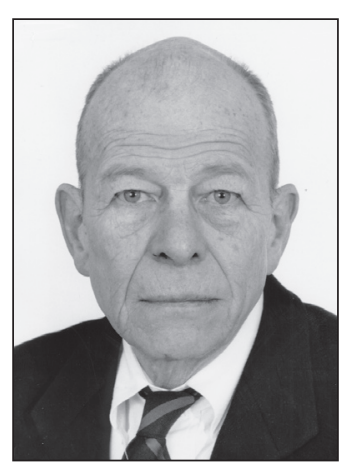

Nick R Anthonisen ans le présent numéro de la Revue canadienne de pneumologie, Rowe et coll. (pages 25-30 [1]) rendent compte des résultats d'une étude prospective portant sur une expérience auprès de 695 adultes asthmatiques à l'urgence de 20 hôpitaux canadiens. Cette étude est digne de mention simplement parce qu'elle a pu être menée jusqu'au bout. Il s'agissait d'une tâche herculéenne.

En résumé, les patients asthmatiques qui se présentaient à l'urgence étaient inclus dans l'étude après avoir donné leur consentement éclairé et avoir rempli des questionnaires normalisés. Leur séjour à l'urgence et un suivi téléphonique étaient consignés. Les patients hospitalisés par suite de those who were not. There were, of course, problems in patient selection because the research team was not available at all times or was not called by the ER physicians, and some patients were too sick to be stabilized enough to give consent, while others were initially believed to have asthma but also had other problems such as pneumonia or chronic obstructive pulmonary disease. Indeed, the 695 patients studied were a subset of approximately 1600 asthma patients seen in participating ERs. While one could whine about the study not being entirely representative, it is difficult to imagine how things could have been improved in a real-world setting.

Of the 695 patients, $13 \%$ were admitted to hospital. If it is assumed that patients who were not stabilized were all admitted, this fraction increased to approximately $18 \%$.

The vast majority of patients were, at least initially, treated successfully. Rowe et al (1) point out that their admission rate was lower than that reported by others in similar studies; however, they wisely refrained from interpreting this to be important because admission rates varied within their study, and it is unlikely that all such studies examine the same type of patients. Approximately one-half of the patients with severe disease (according to a widely accepted acuity scale [2]) were admitted; approximately one-half were not. As one would expect, patients who were admitted were sicker at presentation than those who were not as ill; they were slightly older, more likely to be smokers, had lower oxygen saturations and were on more therapy, especially beta-agonists and oral corticosteroids. They also had longer ER stays than patients who were discharged.

Several 'glitches' in asthma care appeared to emerge from the study. Almost one-third of the patients did not receive bronchodilators during the first hour of their visit; one hopes this figure was at least partially due to poor record keeping as opposed to neglect. Inhaled steroids were underused before the ER visit: $30 \%$ of admitted patients and $40 \%$ of those not admitted had not been receiving them - if someone has the potential to need emergency care, he or she should be taking these medications. The length of the ER stay for admitted patients averaged nearly $11 \mathrm{~h}$, which seems too long; it must have been clear that these patients would have needed admission well before $11 \mathrm{~h}$ had elapsed; this fact may have reflected leur visite à l'urgence étaient comparés à ceux qui ne l'étaient pas. Bien sûr, la sélection des patients posait certains problèmes, car l'équipe de recherche n'était pas disponible en tout temps ou n'était pas appelée par les médecins de l'urgence, et certains patients étaient trop malades pour être suffisamment stabilisés pour donner leur consentement, tandis qu'au départ, on en croyait d'autres asthmatiques alors qu'ils avaient d'autres problèmes, tels qu'une pneumonie ou une maladie pulmonaire obstructive chronique. En fait, les 695 patients à l'étude formaient un sous-groupe d'environ 1600 patients asthmatiques ayant consulté aux urgences participantes. On pourrait se plaindre que l'étude n'ait pas été entièrement représentative, mais il est difficile d'imaginer comment on aurait pu améliorer la situation dans le monde réel.

Des 695 patients, $13 \%$ ont été hospitalisés. Si on postule que les patients dont l'état n'était pas stabilisé ont tous été hospitalisés, cette proportion passe à environ $18 \%$.

La majorité des patients étaient traités avec succès, du moins au départ. Rowe et coll. (1) soulignent que leur taux d'hospitalisation était inférieur à celui déclaré par d'autres dans des études similaires. Toutefois, ils s'abstiennent judicieusement d'interpréter cette constatation comme importante parce que les taux d'hospitalisation variaient dans leur étude, et il est même type de patients. Environ la moitié des patients ayant une maladie grave (selon des échelles d'acuité largement acceptées [2]) ont été hospitalisés, et environ la moitié ne l'ont pas été. Comme on pourrait s'y attendre, les patients hospitalisés étaient plus malades à la présentation que les autres. Ils étaient légèrement plus âgés, plus susceptibles de fumer, avaient une saturation en oxygène plus faible et prenaient plus de traitements, notamment des bêta-agonistes et des corticoïdes oraux. Ils demeuraient également plus longtemps à l'urgence que ceux qui obtenaient leur congé.

Plusieurs «pépins » dans les soins aux patients semblent émerger de l'étude. Environ le tiers des patients n'avaient pas reçu de bronchodilatateurs pendant la première heure de leur dossiers plutôt que de négligence. Les stéroïdes en aérosol étaient peu probable que toutes les études de ce genre portent sur le visite. On espère qu'il s'agit en partie d'une mauvaise tenue des 
bed shortages. The follow-up telephone call two weeks after the ER visit revealed that only one-third of patients had seen their primary care provider during the interval, which is probably bad medicine. Some $15 \%$ of the patients were seen again in the ER within the first two weeks and some were admitted. The obvious and unsurprising conclusion is that best practices are, by no means, universally applied, in spite of well-publicized guidelines. Exactly how much improvement would occur if they had been universally applied is unknown - humans are imperfect and will remain so.

Thus, the study conducted by Rowe et al was descriptive and had substantial power in documenting what actually exists as opposed to what should exist; studies similar to this one should be encouraged. It is worth noting that this group has been very productive $(3,4)$, indicating great energy and dedication.

\section{REFERENCES}

1. Rowe BH, Villa-Roel C, Abu-Laban RB, et al. Admissions to Canadian hospitals for acute asthma: A prospective, multicentre study. Can Respir J 2010;17:25-30.

2. Beveridge R. The Canadian Triage and Acuity Scale: A new and critical element in health care reform. J Emerg Med 1998;16:507-11.

3. Rowe BH, Bopta GW, Clark S, Camargo CA Jr; on behalf the MARC Investigators. Comparison of Canadian versus America emergency department visits for acute asthma. Can Respir J 2007;14:331-7.

4. Rowe BH, Villa-Roel C, Sivilotti M, et al. Relapse following emergency department discharge for acute asthma: A prospective multi-center study. Acad Emerg Med 2008;15:709-17. sous-utilisés avant la visite à l'urgence : $30 \%$ des patients hospitalisés et $40 \%$ de ceux non hospitalisés n'en prenaient pas. Pourtant, quelqu'un qui risque d'avoir besoin de soins d'urgence devrait en prendre. La durée du séjour à l'urgence des patients hospitalisés atteignait une moyenne de près de 11 heures, ce qui semble trop long. Il aurait dû être évident que ces patients avaient besoin d'être hospitalisés bien avant la fin de cette période, qui doit donc refléter une pénurie de lits. L'appel de suivi deux semaines après la visite à l'urgence révélait que seulement le tiers des patients avaient vu leur médecin de soins primaires depuis, ce qui est probablement une mauvaise pratique médicale. Quelque $15 \%$ des patients étaient vus de nouveau à l'urgence pendant ces deux premières semaines, et certains étaient hospitalisés. La conclusion évidente et peu surprenante, c'est que les pratiques exemplaires ne sont en aucun cas appliquées de manière universelle, malgré des lignes directrices bien diffusées. On ne sait quelle aurait été l'amélioration si elles avaient été appliquées de manière universelle. Les humains sont imparfaits et vont le demeurer.

Ainsi, l'étude menée par Rowe et coll. était descriptive et avait le pouvoir substantiel d'attester ce qui existe vraiment plutôt que ce qui devrait exister. Il faudrait encourager des études semblables. Enfin, il convient de souligner que ce groupe s'est montré très productif $(3,4)$, ce qui fait foi d'une grande énergie et d'un grand dévouement.

\section{RÉFÉRENCES}

1. Rowe BH, Villa-Roel C, Abu-Laban RB et coll. Admissions to Canadian hospitals for acute asthma: A prospective, multicentre study. Can Respir J 2010;17:25-30.

2. Beveridge R. The Canadian Triage and Acuity Scale: A new and critical element in health care reform. J Emerg Med 1998;16:507-11.

3. Rowe BH, Bopta GW, Clark S, Camargo CA Jr; on behalf the MARC Investigators. Comparison of Canadian versus America emergency department visits for acute asthma. Can Respir J 2007;14:331-7.

4. Rowe BH, Villa-Roel C, Sivilotti M et coll. Relapse following emergency department discharge for acute asthma: A prospective multi-center study. Acad Emerg Med 2008;15:709-17. 


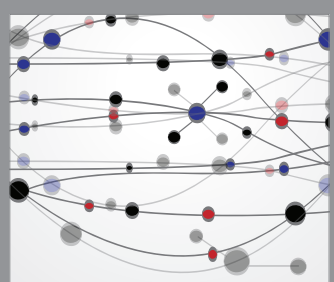

The Scientific World Journal
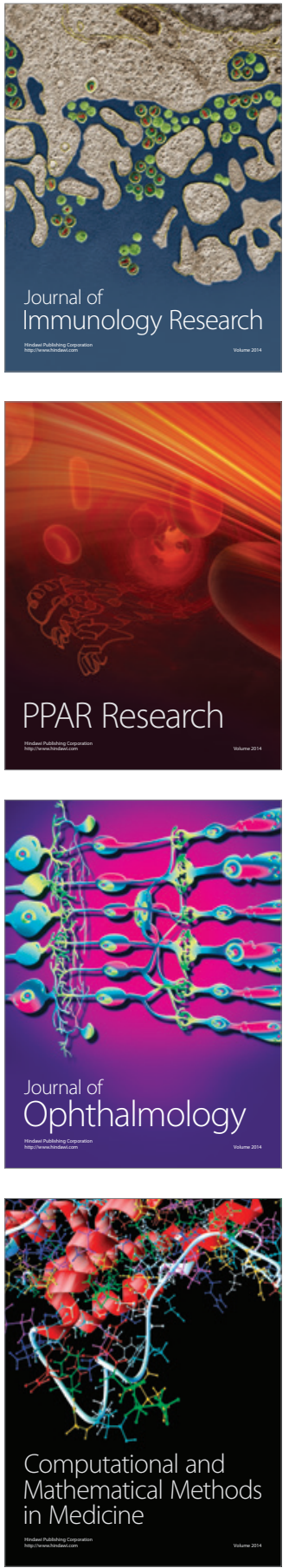

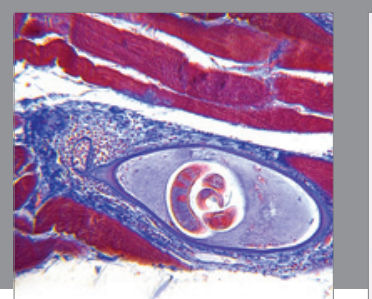

Gastroenterology Research and Practice

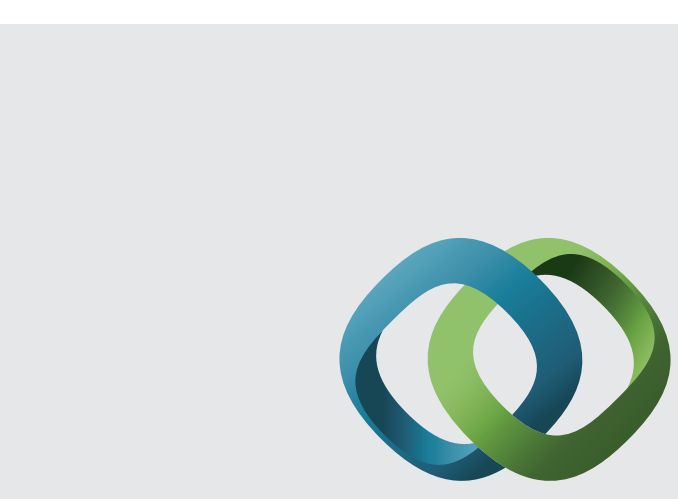

\section{Hindawi}

Submit your manuscripts at

http://www.hindawi.com
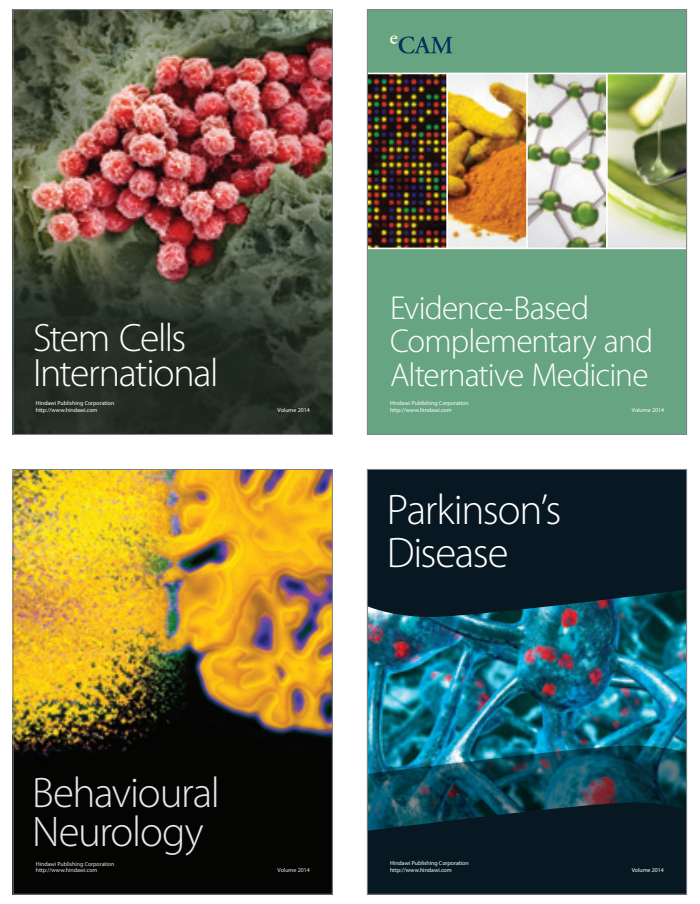
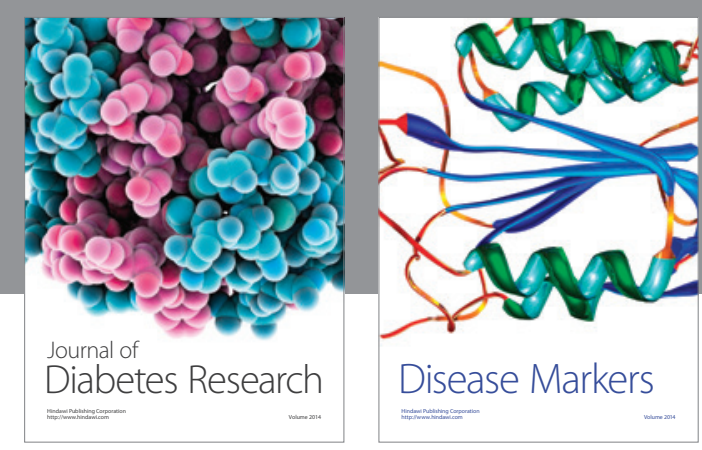

Disease Markers
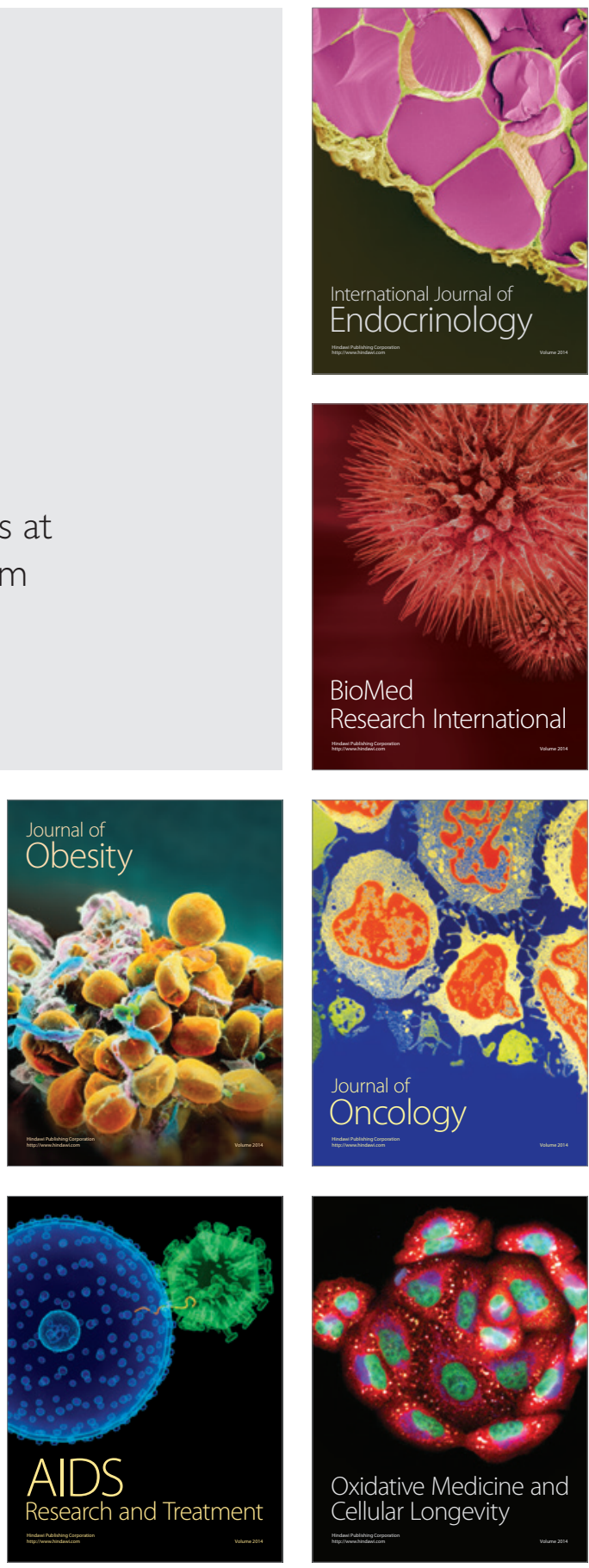